\title{
An improved Monte Carlo technique for radiation shield design
}

\author{
P K SARKAR ${ }^{1}$ and M A PRASAD ${ }^{2}$ \\ ${ }^{1}$ Health Physics Unit, Variable Energy Cyclotron Centre, 1/AF Bidhan \\ Nagar, Calcutta 700064 , India \\ ${ }^{2}$ Division of Radiological Protection, Bhabha Atomic Research Centre, \\ Bombay 400085 , India
}

MS received 6 November 1990

\begin{abstract}
Reduction of statistical error in Monte Carlo shielding calculations is achieved by using antithetic variates with geometrical surface splitting while solving the radiation transport problems. Numerical results are presented for sample calculations for slab shields of different dimensions. The present method shows great improvement in efficiency for thick slabs and therefore is useful for practical shielding calculations.
\end{abstract}

Keywords. Radiation shielding; Monte Carlo calculations; variance reduction; statistical error; antithetic variates; radiation transport.

\section{Introduction}

Monte Carlo calculations are extensively used to solve nuclear engineering problems, e.g., in reactor core design and, more frequently, in the design of complex radiation shields in reactors and particle accelerators. Though the Monte Carlo method is the only practical technique available to solve the Boltzmann transport equation in complicated geometries, statistical errors (variance) are always associated with Monte Carlo results and this error becomes prohibitively large in the case of solving practical problems like calculating transmission through thick shields. Efforts are being directed towards reducing the variance in such calculations by designing and implementing sophisticated variance reduction techniques (Carter \& Cashwell 1977; Hammersley \& Handscomb 1979). Geometrical splitting with Russian roulette is an effective and probably the simplest way of reducing variance in radiation transport calculations. A further reduction of variance is anticipated if geometrical splitting is used in conjunction with antithetic variates.

The method of antithetic variates is one of the most effective variance reduction techniques that can be incorporated in Monte Carlo simulations although it has not become popular with the Monte Carlo practitioners. The name and perhaps the idea of antithetic variates was introduced by Hammersley \& Morton (1956) and has since been expanded by several authors, notably Hammersley \& Mauldon (1956), Handscomb (1958) and Hammersley \& Handscomb (1979). 
In this work we present numerical results using antithetic variates in Monte Carlo radiation transport problems in conjunction with geometric surface splitting and Russian roulette. This is expected to reduce the variance as well as the cost of simulation because antithetic transformations can be incorporated with negligible extra computation cost. Here we estimate the population variance by taking moments around the score. Our approach is based on a method first proposed by Amster \& Djomehri (1976) for estimating population variance associated with analog Monte Carlo games, and later developed to include games using sophisticated variance reduction techniques by Booth \& Amster (1978), Sarkar \& Prasad $(1979,1980,1984)$, Lux (1978, 1983), Booth \& Cashwell (1979), Juzaitis (1982), Dwivedi (1982), Gupta (1983) and Murthy \& Indira (1986). For the present study we have considered two types of antithetic transformations, one proposed by Hammersley \& Morton (1956) and the other by Arvidsen \& Johnsson (1982).

\section{Description of antithetic variates}

The method is based on the construction of a Monte Carlo estimator as a linear combination of other estimators that are negatively correlated. This can be outlined in as below.

If $I_{1}$ and $I_{2}$ are two Monte Carlo estimates of an integral quantity,

$$
I=\int_{0}^{1} g(x) \mathrm{d} x,
$$

then a third estimator $I^{\sim}$ for $I$ can be constructed using a linear combination of $I_{1}$ and $I_{2}$, e.g.,

$$
I^{\sim}=\left(I_{1}+I_{2}\right) / 2 \text {. }
$$

The variance in the estimator $I^{\sim}$ is given by (Carter \& Cashwell 1977)

$$
\operatorname{Var}\left(I^{\sim}\right)=\frac{1}{4} \operatorname{Var}\left(I_{1}\right)+\frac{1}{4} \operatorname{Var}\left(I_{2}\right)+\frac{1}{4} \operatorname{Cov}\left(I_{1}, I_{2}\right) .
$$

If $I_{1}$ and $I_{2}$ are independent, $\operatorname{Cov}\left(I_{1}, I_{2}\right)=0$, but if $I_{1}$ and $I_{2}$ are selected such that they are negatively correlated then $\operatorname{Cov}\left(I_{1}, I_{2}\right) \leqslant 0$, which makes $\operatorname{Var}\left(I^{\sim}\right) \leqslant \operatorname{Var}\left(i_{i}\right)$, $i=1,2$.

Here, however, evaluation of $I^{\sim}$ requires twice as much work as the evaluation of $I_{1}$ or $I_{2}$. Thus one really would like to have $\operatorname{Var}\left(I^{\sim}\right) \leqslant \operatorname{Var}\left(I_{i}\right) / 2$. Carter \& Cashwell (1977) proved that if $g(x)$ is a non-negative monotone non-decreasing (or nonincreasing) function of $x$, then $\operatorname{Var}\left(I^{\sim}\right) \leqslant \operatorname{Var}\left(I_{i}\right) / 2$. Where $g(x)$ is not necessarily monotone, Goertzel \& Kalos (1958) pointed out that an estimator based on the stratification of the interval may be effective, which also had previously been considered by Hammersley \& Morton (1956), and can be described [by denoting $\hat{g}(x)$ as the estimate of $I]$ as follows:

$$
\hat{g}(x)=(1 / n) \sum_{i=1}^{n}\left\{\alpha g\left(\alpha R_{i}\right)+(1-\alpha) g\left[\alpha+(1-\alpha) R_{i}\right]\right\}, \quad \text { for } 0 \leqslant \alpha \leqslant 1,
$$

or defining $G_{\alpha}\left[g\left(R_{i}\right)\right]$ to be the function under the summation,

$$
\hat{g}(x)=(1 / n) \sum_{i=1}^{n} G_{\alpha}\left[g\left(R_{i}\right)\right]
$$


Alternatively,

$$
\hat{g}(x)=(1 / n) \sum_{i=1}^{n}\left\{\alpha g\left(\alpha R_{i}\right)+(1-\alpha) g\left[1-(1-\alpha) R_{i}\right]\right\}, \quad \text { for } 0 \leqslant \alpha \leqslant 1,
$$

or defining $T_{\alpha}\left[g\left(R_{i}\right)\right]$ to be the function under the summation,

$$
\hat{g}(x)=(1 / n) \sum_{i=1}^{n} T_{\alpha}\left[g\left(R_{i}\right)\right]
$$

The transformations $T_{\alpha}$ and $G_{\alpha}$ are linear, preserve expectations and double the number of function evaluations (Sarkar \& Prasad 1989). We intend to incorporate this scheme in Monte Carlo radiation transport with geometrical surface splitting by bringing about a negative correlation among the split particles. As is evident, this scheme can only be applied when the number of split particles are integral powers of 2 i.e., $2^{p}, p=1,2, \ldots$ For $2^{p+1}$ particles the transformation used is $G_{1 / 2}^{p} T_{\alpha}(R)$, where $G_{1 / 2}^{p}$ implies that $G_{1 / 2}$ is operated $p$ times on $T_{\alpha}(R)$, and $\alpha$ is a number between 0 and 1. Examples of generating $R_{k}$ 's and $\alpha_{k}$ 's with this scheme for $p=1$ and $p=2$ are given below:

$p=1$ ( 2 split particles): $R_{1}=\alpha R$ and $R_{2}=[1-(1-\alpha) R]$

$$
\alpha_{1}=\alpha \text { and } \alpha_{2}=(1-\alpha)
$$

$p=2$ (4 split particles): $\mathrm{R}_{1}=(\alpha / 2) R, R_{2}=1 / 2+(\alpha / 2) R$,

$$
\begin{aligned}
& R_{3}=1 / 2-[(1-\alpha) / 2] R, \text { and } R_{4}=1-[(1-\alpha) / 2] R \\
& \alpha_{1}=\alpha_{2}=\alpha / 2, \alpha_{3}=\alpha_{4}=(1-\alpha) / 2
\end{aligned}
$$

We have adopted two schemes of antithetic transformations for relating $R_{k}$ with $R$. Scheme 1, due to Hammersley \& Morton (1956), is the one just described, whereas scheme 2 is given by Arvidsen \& Johnsson (1982) and is as follows.

Scheme 2: In this case for $m$ split particles the $R_{k}$ 's are generated as (denoting $\mathscr{H}(x)$ as the fractional part of $x$ ):

$$
\left.\begin{array}{l}
R_{1}=R, R_{k}=\mathscr{H}\left(2^{k-2} R+1 / 2\right), k=2,3, \ldots m-1 \\
R_{k}=1-\mathscr{H}\left(2^{m-2} R\right), \alpha_{k}=1 / m, k=1,2, \ldots m .
\end{array}\right\} .
$$

It can be seen that for $m=2$, this scheme becomes a special case of scheme 1 with $\alpha=0.5$.

\section{Derivation of the moments equations}

We consider a semi-infinite homogeneous slab of finite length $d$ in the $x$ direction, $0 \leqslant x \leqslant d$, with an isotropic source of particles prescribed either at or near the plane $x=0$. Within the slab there are $N$ surfaces $S_{i}$, parallel to the external faces situated at specified $x=x_{i}^{s}, i=0, \ldots, N-1$. These surfaces are called splitting/Russian roulette surfaces. A particle with weight $w$ moving in the increasing $x$ direction is split at $S_{i}$ into $m_{i}$ identical particles each of weight $w / m_{i}$. Clearly, weight is preserved and we process more particles with smaller weights. In Russian roulette, if a particle proceeds across $S_{i}$ in the direction of decreasing $x$, then the particle is allowed to survive with proabability $1 / m_{i}$ and its weight is increased by a factor $m_{i}$. The particle is killed with a probability $1-\left(1 / m_{i}\right)$. The recipe of splitting and Russian roulette depends mainly 
on the problem being solved. However, a rule-of-thumb (r.t.) splitting is most popularly used where the particles are split 2 for 1 at surfaces $S_{i}$ spaced a distance of one mean free path $(\mathrm{mfp})$ apart.

In deriving the moments equation for transmission of particles through the slab, we denote by $V$ the total space inside the slab and by $V_{i}$ the non-overlapping sub-spaces enclosed by surfaces $S_{i-1}$ and $S_{i}$. Each $V_{i}$ is therefore enclosed by two splitting/Russian roulette surfaces or, by one splitting/Russian roulette surface and one boundary of $V$. In choosing the points of first collision for the $m_{i}$ split particles we use the technique of antithetic variates. To do so we generate the distance of first collision after splitting using a set of numbers $R_{k}\left(0 \leqslant R_{k} \leqslant 1, k=1,2, \ldots, m_{i}\right)$ which are antithetically correlated. This is different from normal geometrical splitting where the histories of the split particles are completely independent of each other. For the present derivations we score particles escaping only through the boundary of $V$ at $x=d$, and consider both the boundaries of $V$ at $x=0$ and at $x=d$ as non-reentrant.

The equations for moments around the score are derived by establishing relationships with points $x$ in $V_{i}$ with others points in $V_{i}$ as well as with points on the boundaries of $V_{i}$. At the points $x_{i}^{S}$ on the splitting/Russian roulette surfaces $S_{i}$ there are discontinuities and we equate the limiting functions on the two sides of these surfaces. The equations derived are for survival biasing and a last event estimator in which a score equal to the weight of the particle is given whenever it crosses the exit boundary of interest. Details of the derivations are reported elsewhere (Sarkar \& Prasad 1989). Here we give equations for the first and second moments around the score and equations for the expected number of flights per history. These equations have been solved numerically to obtain estimates of the variance and expected computation time per history. (Mean number of flights are assumed to be proportional to the mean computation time.)

\subsection{First and second moments around the score}

For transmission through an infinite homogeneous slab of thickness $d$, we restrict ourselves to a one-group problem (constant cross sections) with isotropic scattering. Here $\mu$ is the cosine of the angle between the direction of travel and the normal to the external faces. The integral equation for the first moment around the score is given by

$$
\begin{gathered}
M_{1}(x, \mu)=\left(\Sigma_{s} / 2\right) \int_{x}^{a(\mu)} \mathrm{d} x^{\prime} \int_{-1}^{+1} \mathrm{~d} \mu^{\prime} \exp \left[-\Sigma\left(x^{\prime}-x\right) / \mu\right] / \mu M_{1}\left(x^{\prime}, \mu^{\prime}\right)+ \\
+\Theta(\mu) \exp [-\Sigma(d-x) / \mu],
\end{gathered}
$$

where, $a(\mu)=d \Theta(\mu)$, and $\Theta(\mu)=1$, for $\mu \geqslant 0$, and $\Theta(\mu)=0$, for $\mu<0$. This equation is valid for the pure analogue game also.

For geometrical surface splitting and Russian roulette the equation for the second moment around the score is not continuous across the splitting/Russian roulette surfaces. We therefore have to obtain equations for each $V_{i}$ and establish a relationship between $M_{2}(\cdot)$ 's on either side of the surface. To do so we define the quantities $M_{j}^{-}(\cdot)$ and $M_{j}^{+}(\cdot), j=1,2$, where the minus sign indicates that no surface game has yet been applied and the plus sign denotes that a surface game has just been applied and one of the split (or surviving) particles is ready for a free flight. For the second moment, 
we have

$$
\begin{aligned}
& M_{2}(x, \mu)=\left[\Sigma^{2} /(2 \Sigma)\right] \int_{x}^{b(\mu)} \mathrm{d} x^{\prime} \int_{-1}^{+1} \mathrm{~d} \mu^{\prime} \exp \left[-\Sigma\left(x^{\prime}-x\right) / \mu\right] / \mu M_{2}\left(x^{\prime}, \mu^{\prime}\right)+ \\
& +\exp [-\Sigma(b(\mu)-x) / \mu] M_{2}^{-}\left(x_{i+\Theta(\mu)}, \mu\right) \\
& \quad \text { for } x_{i}^{s}<x<x_{i+1}^{s}, i=0,1, \ldots, N-1,
\end{aligned}
$$

where, $b(\mu)=x_{i+1}^{s} \Theta(\mu)+x_{i}^{s} \Theta(-\mu)$

We note that splitting is resorted to if a particle reaches a splitting/Russian roulette surface $x_{i}^{s}$ with $\mu>0$, and Russian roulette is resorted to if $\mu<0$. For splitting, with antithetic variates we have

$$
\begin{aligned}
M_{2}^{-}\left(x_{i}^{s}, \mu\right)=\sum_{k=1}^{m_{i}} \alpha_{k}^{2} \int_{0}^{1} \mathrm{~d} R M_{2}^{+}\left(x_{i}^{s}, \mu \mid R_{k}\right) & \\
& +2 \sum_{k=1}^{m_{i}} \sum_{k^{\prime}=k+1}^{m_{i}} \alpha_{k} \alpha_{k^{\prime}} \int_{0}^{1} \mathrm{~d} R M_{1}^{+}\left(x_{i}^{s}, \mu \mid R_{k}\right) M_{1}^{+}\left(x_{i}^{s}, \mu \mid R_{k^{\prime}}\right), \\
& \text { for } \mu>0 .
\end{aligned}
$$

Here, $R_{k}$ 's are functions of a single random number $R$ [see (7) and (8)] and $\alpha_{k}$ 's are the corresponding weights satisfying the constraint $\Sigma_{k} \alpha_{k}=1 . M_{j}^{+}\left(x_{i}^{s}, \mu \mid R_{k}\right)$ is the $j$ th moment around the score for particles just split and whose distance to the point of first collision is sampled using the random number $R_{k}$. To evaluate $M_{j}^{+}\left(x_{i}^{s}, \mu \mid R_{k}\right)$ we first define

$$
\left.R_{0}=\exp \left[x_{i+1}^{s}-x_{i}^{s}\right) / \mu\right] .
$$

If $R_{k}<R_{0}$, the particle will reach the next splitting surface at $x_{i+1}^{s}$ without a collision. In that case, we have

$$
M_{j}^{+}\left(x_{i}^{s}, \mu \mid R_{k}\right)=M_{j}^{-}\left(x_{i+1}^{s}, \mu\right), \text { for } \mu>0, j=1,2, R_{k}<R_{0} .
$$

If, on the other hand, $R_{k} \geqslant R_{0}$, the particle will have a collision between $x_{i}^{s}$ and $x_{i+1}^{s}$, where we have

$$
M_{j}^{+}\left(x_{i}^{s}, \mu \mid R_{k}\right)=\frac{1}{2}\left(\Sigma_{s} / \Sigma\right)^{j} \int_{-1}^{1} \mathrm{~d} \mu^{\prime} M_{j}\left(x^{\prime}, \mu^{\prime}\right), \text { for } \mu>0, \quad j=1,2,
$$

with $R_{k} \geqslant R_{0}$ and $x^{\prime}=x-\mu \ln R / \Sigma$.

In the case of ordinary splitting where the split particles are not correlated we have

$$
M_{2}^{-}\left(x_{i}^{s}, \mu\right)=\left(1 / m_{i}\right) M_{2}^{+}\left(x_{i}^{s}, \mu\right)+\left\{\left(m_{i}-1\right) / m_{i}\right\}\left[M_{1}\left(x_{i}^{s}, \mu\right)\right]^{2}
$$

For Russian roulette at $x_{i}^{s}$, we have

$$
M_{2}^{-}\left(x_{i}^{s}, \mu\right)=m_{i} M_{2}^{+}\left(x_{i}^{s}, \mu\right) \text {, for } \mu<0 .
$$

Since we are interested in the transmission through the slab, we use the following scoring scheme-a score of unity is given when a particle of unit weight crosses the 
exit surface at $d$ and a score of 0 if it crosses the exit surface at 0 . We, therefore, have

$$
\begin{array}{ll}
M_{2}^{-}(d, \mu)=1, & \text { for } \mu>0 \\
M_{2}^{-}(0, \mu)=0, & \text { for } \mu<0 .
\end{array}
$$

\subsection{Expected number of flights per history}

As stated previously, we consider the expected number of flights per particle history $N_{i}(\cdot)$, as an indicator of the relative computational time. We briefly describe the scoring system used for this purpose: a score of 1 is given whenever a particle undergoes a collision. A score of 1 is also given if the particle undergoes Russian roulette or splitting, or if it escapes from either of the exit surfaces at 0 and $d$.

The expected number of flights $N_{1}(\cdot)$ is given by

$$
\begin{gathered}
N_{1}(x, \mu)=(\Sigma / 2) \int_{x}^{b(\mu)} \mathrm{d} x^{\prime} \int_{-1}^{+1} \mathrm{~d} \mu^{\prime} \exp \left[-\Sigma\left(x^{\prime}-x\right) / \mu\right] / \mu N_{1}\left(x^{\prime}, \mu^{\prime}\right)+ \\
+\exp [-\Sigma(b(\mu)-x) / \mu]\left[N_{1}^{-}\left(x_{i+\theta(\mu)}^{s}, \mu\right)-1\right]+1,
\end{gathered}
$$

where, for Russian roulette

$$
N_{1}^{-}\left(x_{i}^{s}, \mu\right)=1 / m_{i} N_{1}^{+}\left(x_{i}^{s}, \mu\right)+1, \quad \text { for } \mu<0
$$

and for splitting with antithetic variates

where

$$
N_{1}^{-}\left(x_{i}^{s}, \mu\right)=\sum_{k=1}^{m_{i}} \int_{0}^{1} \mathrm{~d} R N_{1}^{+}\left(x_{i}^{s}, \mu \mid R_{k}\right)+1, \quad \text { for } \mu>0,
$$

$$
\left.\begin{array}{rl}
N_{1}^{+}\left(X_{i}, \mu \mid R_{k}\right) & =N_{1}^{-}\left(X_{i+1}, \mu \mid R_{k}\right) \text { for } R_{k}<R_{0} \\
& =1 / 2 \int_{-1}^{1} \mathrm{~d} \mu^{\prime} N_{1}\left(x^{\prime}, \mu^{\prime}\right)+1, \text { for } R_{k} \geqslant R_{0},
\end{array}\right\},
$$

with $x^{\prime}=x-\mu \ln R_{k} / \Sigma$. For ordinary splitting we have.

$$
N_{1}^{-}\left(x_{i}^{s}, \mu\right)=m_{i} N_{1}^{+}\left(x_{i}^{s}, \mu\right)+1 .
$$

We give a score of unity in this case whenever the particle escapes through any one of the exit boundaries. Thus, for the exit surfaces

$$
\left.\begin{array}{ll}
N_{1}^{-}(\mathrm{d}, \mu)=1, & \text { for } \mu>0 \\
N_{1}^{-}(0, \mu)=1, & \text { for } \mu<0
\end{array}\right\}
$$

\section{Numerical results and discussions}

The coupled set of integral equations for the first and second moments of the transmission score as well as for the mean number of flights were solved iteratively as in our previous work (Sarkar \& Prasad 1979, 1980, 1984) for a number of slab thickness and scattering probabilities. In every case, splitting was carried out at the source point also. 
Table 1. Comparison of Monte Carlo (MC) results with deterministic solutions of integral equations (IE) in the case of rule-of-thumb splitting for a 5-mfp slab The antithetic variates scheme is due to Arvidsen \& Johnsson (1982).

\begin{tabular}{|c|c|c|c|c|c|}
\hline \multirow[b]{2}{*}{$\Sigma_{s} / \Sigma$} & & \multicolumn{2}{|c|}{ Ordinary splitting } & \multicolumn{2}{|c|}{$\begin{array}{l}\text { Splitting with antithetic } \\
\text { variates (scheme } 2 \text { ) }\end{array}$} \\
\hline & & Variance & $\begin{array}{l}\text { No. of } \\
\text { flights }\end{array}$ & Variance & $\begin{array}{l}\text { No. of } \\
\text { flights }\end{array}$ \\
\hline \multirow[t]{2}{*}{0.9} & IE & $0.51(-2)$ & $142 \cdot 0$ & $0 \cdot 30(-2)$ & $142 \cdot 0$ \\
\hline & $\mathrm{MC}$ & $0.60(-2)$ & $140 \cdot 0$ & $0.25(-2)$ & $139 \cdot 0$ \\
\hline \multirow[t]{2}{*}{0.5} & IE & $0.57(-3)$ & $142 \cdot 0$ & $0 \cdot 24(-3)$ & $142 \cdot 0$ \\
\hline & $\mathrm{MC}$ & $0 \cdot 51(-3)$ & $144 \cdot 0$ & $0 \cdot 20(-3)$ & $141 \cdot 0$ \\
\hline \multirow[t]{2}{*}{$0 \cdot 1$} & IE & $0 \cdot 36(-3)$ & $142 \cdot 0$ & $0 \cdot 15(-3)$ & $142 \cdot 0$ \\
\hline & $\mathrm{MC}$ & $0.40(-3)$ & $141 \cdot 0$ & $0.11(-3)$ & $140 \cdot 0$ \\
\hline
\end{tabular}

As a check on these calculations, results were also obtained from actual Monte Carlo programs and the two sets of values compared. Table 1 gives the comparative results for a $5 \mathrm{mfp}$ slab with 3 different scattering probabilities $(0 \cdot 9,0 \cdot 5,0 \cdot 1)$. Survival biasing and rule of thumb splitting (splitting in the ratio $1: 2$ at every mean free path) were used. The Monte Carlo results are for 50,000 histories. The agreement may be considered satisfactory given the statistical variations in the Monte Carlo method.

Table 2 gives the population variance, number of flights per history and the relative efficiency for rule of thumb splitting for slabs of 3,510 and $20 \mathrm{mfp}$ thickness for different scattering probabilities $(0 \cdot 1,0 \cdot 5,0 \cdot 9)$. We have considered the quantity $1 /$ (variance per history $x$ number of flights per history) as the efficiency. Relative efficiency is defined as the ratio of the efficiencies of a transmission scheme using splitting with antithetic transformations and the same transmission scheme using ordinary splitting. For example, the relative efficiencies for all the schemes in table 2 using ordinary splitting is 1 . We can see from the results that we get very good improvement in efficiency for low scattering cross sections using anithetic transformations, especially with scheme 1 (Hammersley \& Morton 1956). The relative gain in efficiency increases with decrease in scattering probability and also with increasing thickness of the slab. This is understandable because with decrease in scattering probability (i.e. more absorption) as well as with the increase in the thickness of the slab, the function representing the first moment or the expected transmission score $M_{1}(x)$ becomes steeper with respect to $x$. It is also observed that the gain in efficiency is more for scheme 1 (Hammersley \& Morton 1956) than that for scheme 2 (Arvidsen \& Johnsson 1982). This is because in the scheme suggested by Hammersley and Morton, in addition to antithetic transformations, some amount of stratification is also done.

It may be noted at this point that the values for scheme 1 are given at the optimal $\alpha$. As stated earlier the optimal values of $\alpha^{\prime}$ s are obtained by numerically estimating the efficiencies with different $\alpha$ values. Table 3 gives, for r.t. splitting with survival biasing, the values of population variance, number of flights and the product of the two for 5, 10 and $20 \mathrm{mfp}$ with different scattering probabilities with respect to different $\alpha$ values. It may be noted here that because of survival biasing the number of flights for a fixed slab thicknes is independent of the scattering probability. It is observed 


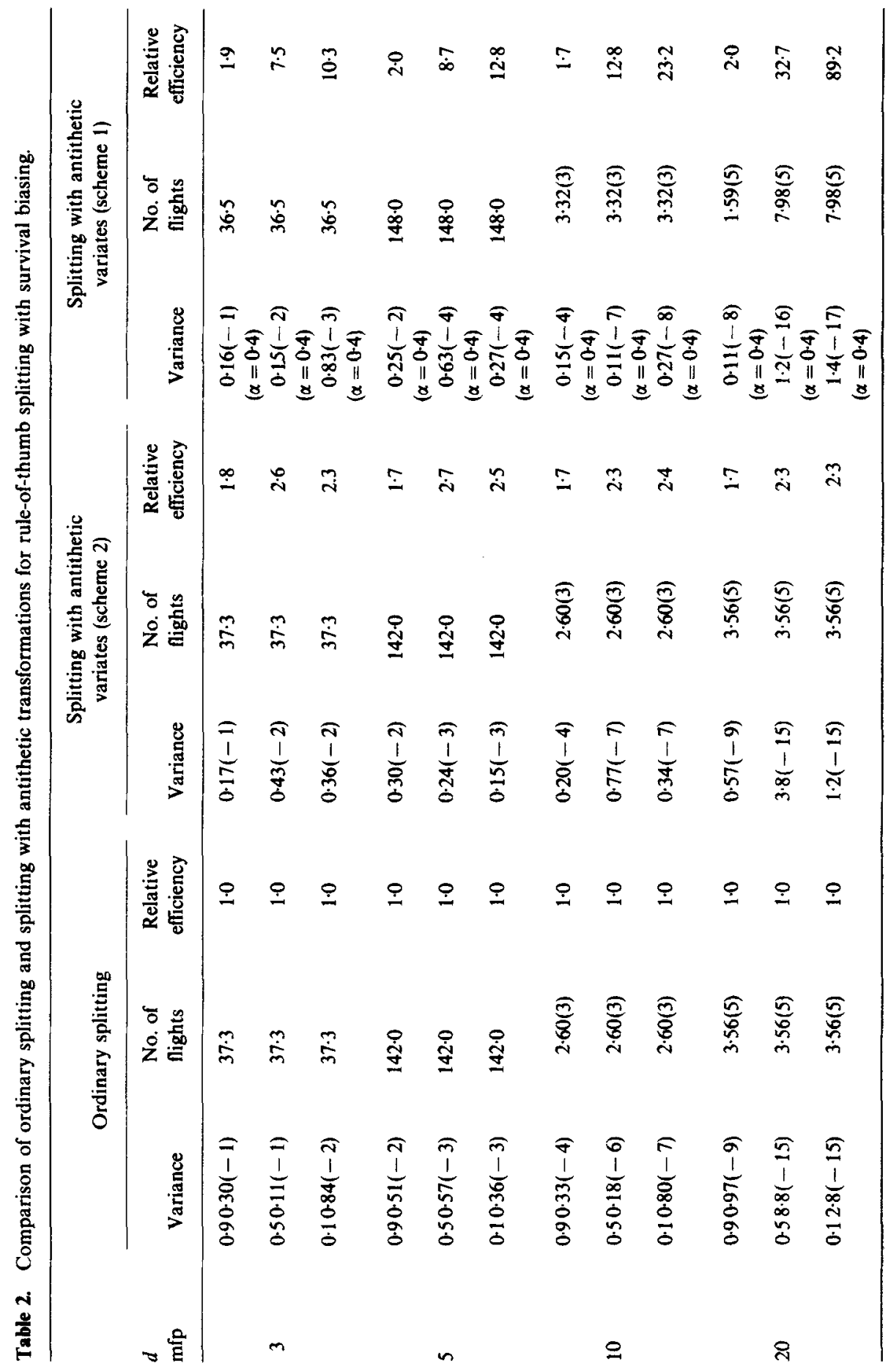


Table 3. Variance and efficiencies for different values of $\alpha$ for rule-of-thumb splitting with survival biasing. Antithetic variates used are due to Hammersley \& Morion (1956).

\begin{tabular}{|c|c|c|c|c|c|c|c|c|}
\hline \multirow[b]{2}{*}{$\begin{array}{l}d \\
\mathrm{mfp}\end{array}$} & \multirow[b]{2}{*}{$\alpha$} & \multirow{2}{*}{$\begin{array}{c}\text { Number } \\
\text { of } \\
\text { flights } \\
N_{1}\end{array}$} & \multicolumn{2}{|c|}{$\Sigma_{s} / \Sigma=0.9$} & \multicolumn{2}{|c|}{$\Sigma_{s} / \Sigma=0.5$} & \multicolumn{2}{|c|}{$\Sigma_{s} / \Sigma=0 \cdot 1$} \\
\hline & & & $\begin{array}{l}\text { Variance } \\
\qquad \sigma^{2}\end{array}$ & $N_{1} \times \sigma^{2}$ & $\begin{array}{l}\text { Variance } \\
\qquad \sigma^{2}\end{array}$ & $N_{1} \times \sigma^{2}$ & $\begin{array}{l}\text { Variance } \\
\qquad \sigma^{2}\end{array}$ & $N_{1} \times \sigma^{2}$ \\
\hline \multirow{5}{*}{5} & 0.2 & $1 \cdot 38(+2)$ & $7 \cdot 7(-3)$ & $1 \cdot 1(+0)$ & $4 \cdot 6(-4)$ & $6 \cdot 3(-2)$ & $2 \cdot 6(-4)$ & $3 \cdot 6(-2)$ \\
\hline & 0.3 & $1 \cdot 46(+2)$ & $3 \cdot 8(-3)$ & $5 \cdot 5(-1)$ & $1 \cdot 3(-4)$ & $1 \cdot 9(-2)$ & $5 \cdot 7(-5)$ & $8-3(-2)$ \\
\hline & 0.4 & $1.48(+2)$ & $2 \cdot 5(-3)$ & $3 \cdot 7(-1)$ & $6 \cdot 3(-5)$ & $9 \cdot 3(-3)$ & $2 \cdot 7(-5)$ & $4 \cdot 0(-3)$ \\
\hline & 0.5 & $1.42(+2)$ & $3 \cdot 0(-3)$ & $4 \cdot 3(-1)$ & $2 \cdot 4(-4)$ & $3 \cdot 4(-2)$ & $1 \cdot 5(-4)$ & $2 \cdot 1(-2)$ \\
\hline & 0.6 & $1 \cdot 30(+2)$ & $4 \cdot 4(-3)$ & $5 \cdot 7(-1)$ & $6 \cdot 0(-4)$ & $7 \cdot 8(-2)$ & $4 \cdot 3(-4)$ & $5 \cdot 6(-2)$ \\
\hline \multirow{5}{*}{10} & 0.2 & $4 \cdot 07(+3)$ & $6 \cdot 0(-5)$ & $2 \cdot 4(-1)$ & $1 \cdot 2(-7)$ & $4 \cdot 9(-4)$ & $4 \cdot 4(-8)$ & $1 \cdot 8(-4)$ \\
\hline & $0 \cdot 3$ & $3.97(+3)$ & $2 \cdot 3(-5)$ & $9 \cdot 1(-2)$ & $1 \cdot 8(-8)$ & $7 \cdot 1(-5)$ & $4 \cdot 4(-9)$ & $1 \cdot 7(-5)$ \\
\hline & 0.4 & $3 \cdot 32(+2)$ & $1 \cdot 5(-5)$ & $5 \cdot 0(-2)$ & $1 \cdot 1(-8)$ & $3 \cdot 6(-6)$ & $2 \cdot 7(-9)$ & $8 \cdot 9(-7)$ \\
\hline & 0.5 & $2 \cdot 60(+3)$ & $2 \cdot 0(-5)$ & $5 \cdot 2(-2)$ & $7 \cdot 7(-8)$ & $2 \cdot 0(-4)$ & $3 \cdot 5(-8)$ & $9 \cdot 1(-5)$ \\
\hline & 0.6 & $1.95(+3)$ & $3 \cdot 4(-5)$ & $6 \cdot 6(-2)$ & $3 \cdot 8(-7)$ & $7 \cdot 4(-4)$ & $2 \cdot 2(-7)$ & $3 \cdot 6(-4)$ \\
\hline \multirow{5}{*}{20} & 0.2 & $2 \cdot 65(+6)$ & $2 \cdot 0(-10)$ & $6 \cdot 9(-1)$ & $3 \cdot 5(-15)$ & $9 \cdot 3(-9)$ & $7 \cdot 9(-16)$ & $2 \cdot 1(-9)$ \\
\hline & 0.3 & $1 \cdot 77(+6)$ & $6 \cdot 7(-10)$ & $1 \cdot 2(-3)$ & $1.5(-16)$ & $2 \cdot 7(-10)$ & $1 \cdot 3(-17)$ & $2 \cdot 3(-11)$ \\
\hline & 0.4 & $7 \cdot 98(+5)$ & $4 \cdot 4(-10)$ & $3 \cdot 5(-4)$ & $1 \cdot 2(-16)$ & $9 \cdot 6(-11)$ & $1 \cdot 4(-17)$ & $1 \cdot 1(-11)$ \\
\hline & 0.5 & $3 \cdot 56(+5)$ & $5 \cdot 7(-10)$ & $2 \cdot 0(-4)$ & $3 \cdot 8(-15)$ & $1 \cdot 4(-9)$ & $1 \cdot 2(-15)$ & $4 \cdot 3(-11)$ \\
\hline & 0.6 & $1 \cdot 59(+5)$ & $1 \cdot 1(-9)$ & $1 \cdot 7(-4)$ & $9 \cdot 6(-14)$ & $1 \cdot 5(-9)$ & $4 \cdot 6(-14)$ & $7 \cdot 3(-9)$ \\
\hline
\end{tabular}

from the results that for slab thickness of 5 and $10 \mathrm{mfp}$ the optimal value of $\alpha$ is 0.4 both for minimum variance and maximum efficiency at all values of the scattering probability. For a slab thickness of $20 \mathrm{mfp}$, however, optimal values of $\alpha$ with regard to maximum efficiency occur at 0.6 for $\Sigma_{s} / \Sigma=0.9$, for other scattering probabilities it remains at 0.4 . Nevertheless, minimum variance is observed around $\alpha=0.4$ for all scattering probabilities. The variance increases rather sharply on both sides of the optimal value, especially for low scattering probabilities. At $\alpha=0.5$ schemes 1 and 2 become identical. The sharp rise in variance around $\alpha=0.4$ in r.t. splitting explains the appreciable increase in gain for scheme 1 over scheme 2 , for low scattering probabilities. It is quite plausible that the reduction in variance for scheme 1 is mostly due to stratification of the interval. The effect of the negative correlation between the collision points for r.t. splitting results in an increase in the efficiency by a factor that ranges between $1 \cdot 7$ and 2.5 .

\section{Conclusions}

The following conclusions may be drawn for Monte Carlo shielding calculations on the basis of these results.

(1) Antithetic variates in conjunction with geometric surface splitting always leads to reduction in variance and increase in efficiency in comparison to ordinary splitting provided optimal antithetic parameters are chosen.

(2) A substantial gain in efficiency is obtained by incorporating antithetic transformations in rule of thumb splitting. The advantage gained for thick slabs with low scattering probabilities is attractively large. This indicates the usefulness of the present method in practical shielding calculations since radiation shields are thick and are constructed with materials that have low scattering and high absorption cross-sections. 


\section{References}

Amster H J, Djomehri M J 1976 Nucl. Sci. Eny. 60: 131-141

Arvidsen N I, Johnsson T 1982 J. Stat. Comput. Simulation 15: 119-125

Booth T E, Amster H J 1978 Nucl. Sci. Eng. 65: 273-280

Booth T E, Cashwell E D 1979 Nucl. Sci. Eng. 71: 128-142

Carter L L, Cashwell E D 1977 Particle transport simulation with the Monte Carlo method (Oak Ridge: Tech. Inf. Centre, Energy, Res. Dev. Admin.)

Dwivedi S R 1982 Nucl. Sci. Eng. 80: 172-178

Goertzel G, Kalos M H 1958 Monte Carlo methods in transport problems. Progress in Nuclear Science, series 1 (New York: Pergamon) vol. 2

Gupta H C 1983 Nucl. Sci. Eng. 83: 187-197

Hammersley J M, Handscomb D C 1979 Monte Carlo methods (London: Chapman and Hall)

Hammersley J M, Mauldon J G 1956 Proc. Cambridge Philos. Soc. 52: 476-481

Hammersley J M, Morton K W 1956 Proc. Cambridge Philos. Soc. 52: 449-475

Handscomb D C 1958 Proc. Cambridge Philos. Soc. 54: 300-301

Juzaitis R J 1982 Nucl. Sci. Eng. 80: 424-447

Lux I 1978 Nucl. Sci. Eng. 67: 317-325

Lux I 1983 Nucl. Sci. Eng. 83: 198-205

Murthy K P N, Indira R 1986 Nucl. Sci. Eng. 92: 482-487

Sarkar P K. Prasad M A 1979 Nucl. Sci. Eng. 70: 243-261

Sarkar P K, Prasad M A 1980 Nucl. Sci. Eng. 74: 52-60

Sarkar P K, Prasad M A 1984 Nucl. Sci. Eng. 87: 136-151

Sarkar P K, Prasad M A 1989 A numerical analysis of antithetic variates in Monte Carlo radiation transport with geometrical surface splitting, Report, BARC-1493, Bhabha Atomic Research Centre, Bombay 